\title{
Hyperspectral imaging technique for offal quantification in minced meat
}

\author{
M. Kamruzzaman ${ }^{1}$, M. E. Haque ${ }^{2^{\star}}$ and M. R. Ali ${ }^{3}$ \\ ${ }^{1}$ Department of Food Technology and Rural Industries, ${ }^{2}$ Department of Computer Science \& Mathematics and \\ ${ }^{3}$ Department of Farm Power and Machinery, Bangladesh Agricultural University, Mymensingh-2202, Bangladesh, \\ *E-mail : enamul@bau.edu.bd
}

\begin{abstract}
Spectral imaging is a new technique that combines conventional imaging and spectroscopy in a single system to obtain both spatial and spectral information simultaneously from an object. In this study, potential of hyperspectral imaging in the spectral range of 910-1700 $\mathrm{nm}$ was investigated for detecting adulteration in minced lamb meat. Spectral data were extracted to develop a partial least squares regression (PLSR) model to predict the level of adulteration in minced lamb. Good prediction model was obtained using the whole spectral range with a coefficient of determination $\left(R^{2} \mathrm{CV}\right)$ of 0.97 and root-mean-square errors estimated by cross validation (RMSECV) of $1.80 \%$. Successive projection algorithm (SPA) was employed for optimal waveband selection. The PLSR model using only 7 optimum wavelengths $(930,1067,1396,1460,1658,1668$, and $1702 \mathrm{~nm})$ resulted in a coefficient of determination $\left(\mathrm{R}^{2} \mathrm{CV}\right)$ of 0.97 and RMSECV of $1.84 \%$. The study demonstrated the ability of the hyperspectral imaging as a rapid and alternative to the time-consuming and conventional methods to detect adulteration in minced lamb meat.
\end{abstract}

Keywords: Adulteration, Hyperspectral imaging, Wavelength Selection, Minced meat, Offal, PLSR

\section{Introduction}

Meat products play important role in our daily meals by providing protein for helping body movement and metabolism. But, adulterations of meat products mixing with cheaper substances (such as animal offal, flour and gelatin) were frequently reported (Wu et al. 2013, Morsy and Sun 2013) and thus pose a great concern to consumers. Such adulteration is not only a commercial malpractice but also a health risk as certain consumers may be allergic to the adulterant material (Zhao et al., 2014). Several methods such as chromatography, electrophoretic separation of proteins, immunological procedure and DNA based techniques have been utilized for qualitative and/or quantitative analysis of adulteration for authentication of minced meat. These techniques are often not suitable for a fast-paced processing environment because these methods are slow, destructive and also require sophisticated laboratory procedures with monotonous sample preparation. Consequently, a rapid, reliable and non-destructive method is required. Recently, hyperspectral imaging (HSI) has emerged as a rapid and effective tool for non-destructive inspection of various agro- food products. The HSI technology was originally developed by NASA in the field of remote sensing (Goetz et al., 1985). However, the technology is now passing the point of scientific curiosity and is currently under active evaluation by industry and academia in many different applications (EIMasry et al., 2012). This technology mingles the advantages of both spectroscopy and imaging techniques in one system to provide both spectral and spatial information for better inspection. The power of spectroscopy is used to detect or quantify chemical constituents based on their spectral signature and imaging transforms this information into chemical maps in the form of concentration profiles. Recently, this technique has received a considerable attention for assessing fruits (ElMasry et al., 2008), vegetables (Ariana and Lu, 2010), red meat (Naganathan et al., 2008, Qiao et al., 2007), poultry (Nakariyakul and Casasent, 2008), Turkey (Iqbal et al., 2013), fish (Grau et al., 2011; Segtnan, et al., 2009; Sone et al., 2012). However, only one study reported the use hyperspectral imaging to quantify the level of adulteration i.e. pork in minced lamb meat (Kamruzzaman et al., 2013). The objective of this research is to utilize hyperspectral imaging coupling with PLSR analysis for predicting the adulteration level (i.e. heart) in minced lamb meat. 


\section{Materials and Methods}

\section{Sample preparation}

Minced lamb meat and lamb heart were purchased from local retail stores and transported to the laboratory of Biosystems Engineering, University College Dublin (UCD) for image acquisition. Firstly, lamb heart was cut into small pieces and then fully minced. Then, the minced lamb was weighed and evenly mixed with minced heart at a certain proportion to guarantee the adulteration percentage ranged from $2 \%$ to $36 \%$ with around $2 \%$ increment. Finally, the mixture was placed on a round metal lid for image acquisition. One sample for each adulteration level was prepared. Therefore, a total number of 18 samples were used in the study.

\section{Imaging acquisition and correction}

The line-scanned hyperspectral imaging system in the reflectance mode was used in the experiment as shown in Fig. 1. The imaging unit was composed of a 12-bit CCD camera, a high performance spectrograph (ImSpector N17E, Specim, Spectral Imaging Ltd., Finland) ), an illumination unit of two 500W tungsten halogen lamps (V-light, Lowel Light Inc, USA), a translation stage (MSA15R-N, AMTLinearways, SuperSlides \& Bushes Corp., India), a data acquisition software (SpectralCube, Spectral Imaging Ltd., Finland). Each sample was placed on the translation stage and a hyperspectral image of the sample was acquired line by line. The acquired images were stored in a raw format before being processed. The image acquisition process was controlled by the SpectralCube data acquisition software (Spectral Imaging Ltd., Finland). Each image was recorded in the NIR region of 910-1700 nm with a spectral increment of about $3.34 \mathrm{~nm}$ between the contiguous bands, thus producing a total of 237 bands. After image acquisition, reflectance calibration was firstly performed on the obtained hyperspectral images of adulterated minced lamb using white/dark reference, as described by Kamruzzaman et al., (2013).

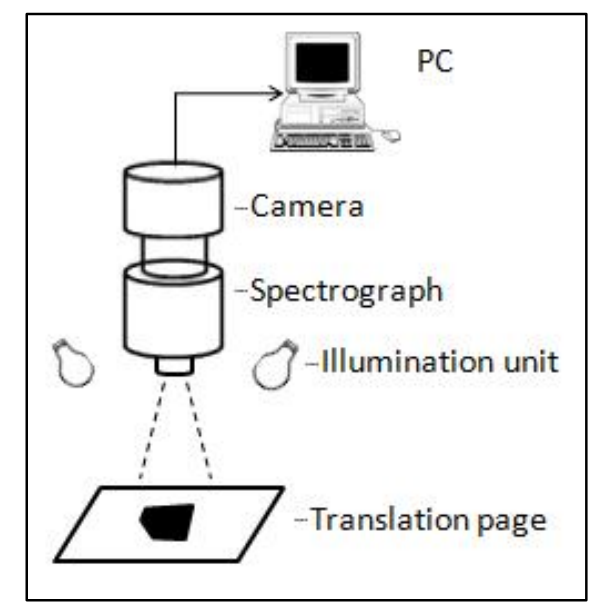

Fig.1. Components of a line-scan hyperspectral imaging system

\section{Extraction of spatial data}

Spectral data were extracted from the minced meat by ignoring background of the sample. A binary mask image was created by thresholding the image at $1300 \mathrm{~nm}$ with a value of 0.25 . The mask was used as the main region of interest (ROI) to extract spectral data from the calibrated hyperspectral image. The reflectance spectrum from the ROI was computed by averaging the spectral value of all pixels in the ROI to produce only one mean spectrum for each sample. The same procedure was repeated to obtain the spectrum for all the tested samples. The extracted spectral data of each subsample were then arranged in spectral matrix $(X)$ where the columns of this matrix represent the wavelengths (237 variables) and the rows of this matrix represent samples (18 samples). 


\section{Data analysis}

Partial least squared regression (PLSR) was applied on the full-wavelength range (910-1700 nm) for calibration and leave-one-out cross-validation (LOOCV). Partial least squares regression (PLSR) is a method for constructing predictive models where the factors are many in number and highly collinear. The core emphasis of this method is to predict the responses rather than identifying the underlying relationship between variables. PLSR method employs linear algorithm as there is a liner relationship between spectra and object properties (Kamruzzaman et al., 2013). Thus PLSR builds a liner model to predict a set of dependent variables, y (i.e. concentration of chemical attributes) from a large set of independent variables, $X$ (i.e. predictors or wavelengths). Among many variables, there may be only a few underlying or latent factors that account for best predictive response (Wu et al., 2013). These orthogonal factors are called latent variables (LVs). These LVs are designed appropriately to capture most information in $\mathrm{X}$ as well as $\mathrm{y}$. The PLSR analysis was carried out using the Unscrambler software v9.5 (CAMO AS, Trondheim, Norway). Performance of the regression models was evaluated using the root mean square errors of calibration (RMSEC), the coefficient of determination in calibration $\left(R^{2}{ }_{c}\right)$, the root mean square errors estimated by cross-validation (RMSECV), and the coefficient of determination in cross-validation $\left(\mathrm{R}_{\mathrm{CV}}^{2}\right)$.

\section{Optimum wavelengths selection}

To reduce data redundancy and speed up analysis, the most pertinent wavebands were selected using successive projection algorithm. Successive projections algorithm (SPA) was proposed in (Araújo et al., 2001) for the optimum wavelengths selection. This method is a novel variable selection algorithm designed to solve the co-linearity problems by selecting variables with minimal redundancy. Nowadays SPA method has found its way with different modelling (Wu et al., 2012). Basically, SPA comprises three main stages. Initially, the algorithm builds candidate subsets of variables with minimum colinearity as the result of projection operations applied to the columns of the spectral matrix available for the training data. The second stage involves the evaluation of candidate subsets of variables based on the value of root mean square error (RMSE) obtained from the resulting multi linear regression (MLR) model assessed by cross-validation or by applying a separate validation set. The final stage consists of a variable elimination procedure aimed at removing uninformative variables without significant loss of prediction ability. Though SPA's simple projection operations is quite effective, there are some drawbacks also. Variables selected by SPA may have a low signal-to-noise ratio $(\mathrm{S} / \mathrm{N})$ or be insufficient for multivariate calibration which can affect the precision of model prediction. When the developed model is optimized by selecting several important wavelengths/variables, the spectra can be applied in a pixel by pixel manner to obtain the distribution map where the components of the samples are clearly visualized and easily interpretable. The distribution map can be visually improved by employing post-processing routines.

\section{Results and Discussion}

\section{Spectral features of heart adulteration}

The mean relative reflectance spectra representing different heart adulteration levels in the minced lamb meat are shown in Fig. 2. Although the spectral curves show a similar pattern for different adulteration levels, the reflectance values throughout the entire spectral regions decreased with increasing adulteration percentage, indicating a close relationship between the adulteration content and the reflectance spectra. In the spectra, the major absorption bands were observed at 974, 1211 and 1440 $\mathrm{nm}$. Absorption peaks identified at $974 \mathrm{~nm}$ and $1440 \mathrm{~nm}$ are due to water absorption bands related to O$\mathrm{H}$ stretching second and first overtones respectively (Barlocco et al., 2006), and absorption bands around $1211 \mathrm{~nm}$ are related to $\mathrm{C}-\mathrm{H}$ stretching second overtone which is attributed to fat content (Andrés et al., 2008). 


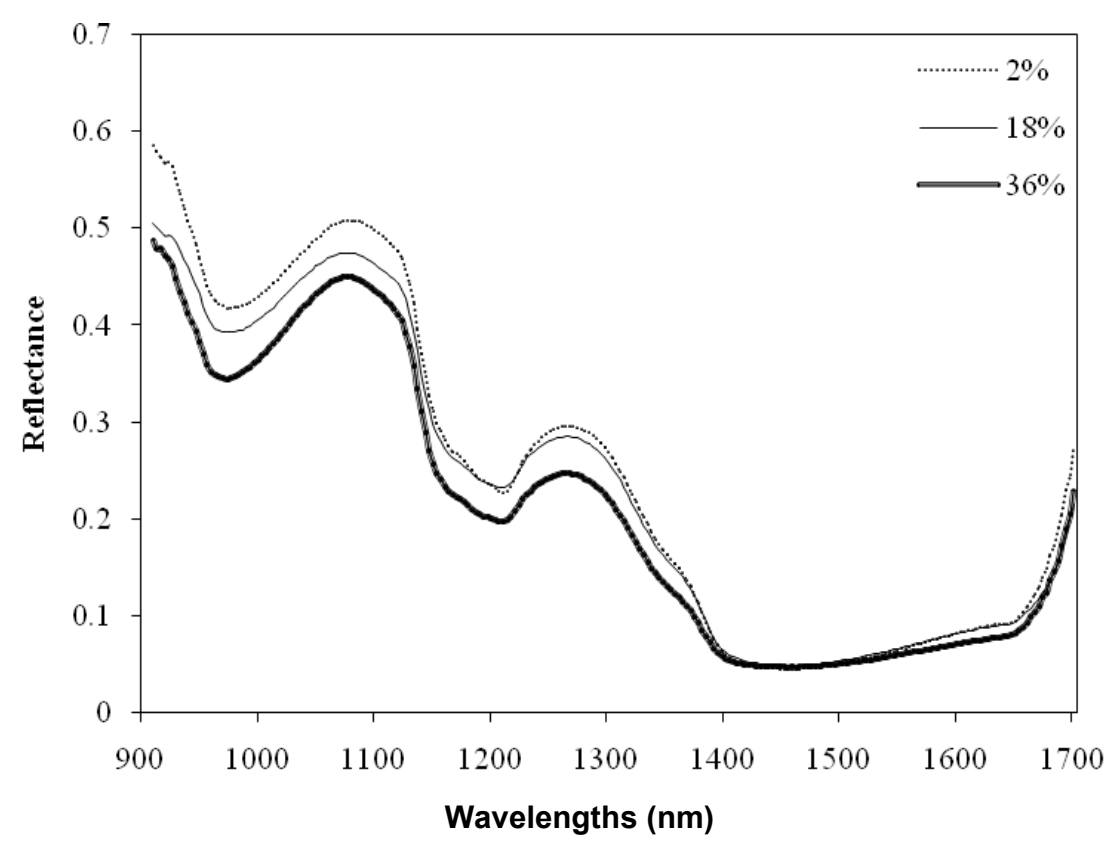

Fig. 2. The mean NIR spectra of different adulteration percentage extracted from ROI in hyperspectral images for heart adulteration

\section{PLSR model development with full wavelength range $(900-1700 \mathrm{~nm})$}

Regression model was developed using PLSR to correlate the spectral data with the level of adulterations. Full cross validation was utilized to validate the calibration model. The critical step for an accurate PLSR model is to select the correct number of latent variables (LVs) needed to obtain the best prediction. In this study, it was selected at the lowest value of predicted residual error sum of squares (PRESS). PLSR prediction results are presented in the scatter plots shown in Fig. 3. It was possible to detect adulteration in minced lamb with high performance in both calibration and cross-validation conditions using five LVs. The level of adulteration in minced lamb was predicted with a coefficient of determination in calibration $\left(R^{2}\right.$ ) of 0.99 , standard error of calibration (RMSEC) of $1.21 \%$, coefficient of determination in cross-validation $\left(\mathrm{R}^{2} \mathrm{CV}\right)$ of 0.97 and standard error from cross-validation (RMSECV) of $1.80 \%$. The model had good prediction ability as accentuated by the high values of the coefficients of determination as well as the small difference between RMSEC and RMSECV. The results obtained in this study were higher than those reported by Ding \& Xu (2000) but were similar to those mentioned by MezaMárquez et al. (2010) for predicting adulteration in minced beef using NIR spectroscopy. Meza-Márquez et al. (2010) reported $\mathrm{R}_{\mathrm{p}}^{2}$ of 0.99 for quantifying horse adulteration in minced beef, whereas Ding \& Xu (2000) obtained lower values of $R^{2}$ in predicting adulteration in beef hamburgers with mutton $\left(R_{c v}^{2}=0.86\right)$ and pork $\left(R^{2}{ }_{c v}=0.89\right)$.

\section{Optimum wavelength selection}

Wavelength selection is a critical step in spectral data analysis to reduce the high dimensionality of spectral data. In essence, the selection of informative variables could produce better prediction and simpler models. In this study, SPA was used to select the optimum wavelengths. The technique has been used to solve the co-linearity problems by selecting variables with minimal redundancy with different modelling techniques such as SPA-PLS, SPA-least square support vector machine (LS-SVM) SPAartificial neural network (ANN), SPA-linear discriminate analysis (LDA) ( Wu et al., 2012). By using SPA method, seven wavelengths $(930,1067,1396,1460,1658,1668$, and $1702 \mathrm{~nm})$ were selected as optimum wavelengths for detecting adulteration in minced lamb as shown in Fig. 4. The selected optimum wavelengths are indicated by circles on top of a representative spectrum in Fig. 4. 


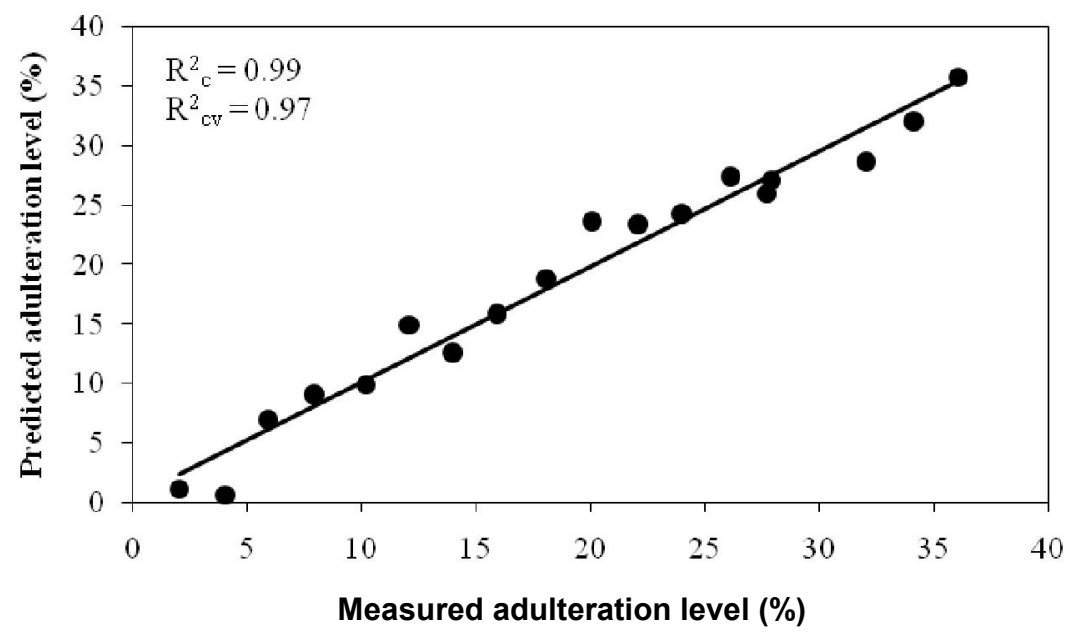

Fig.3. Predicted versus measured adulteration levels of tested samples using PLSR model at full wavelengths range

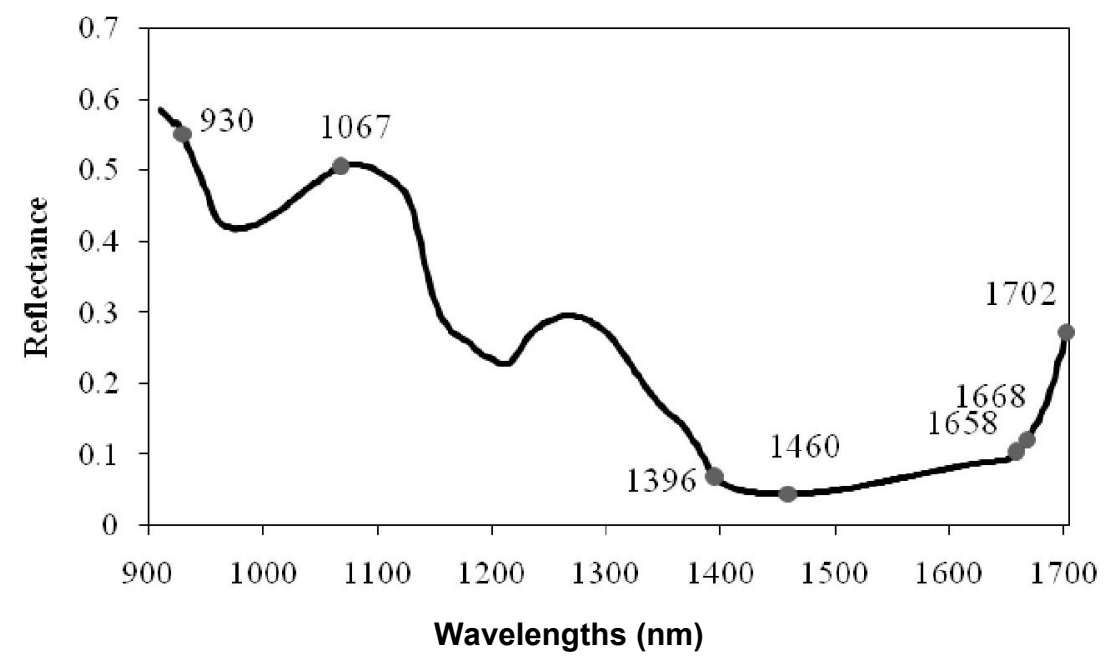

Fig.4. Selection of optimum wavelengths by SPA for predicting adulteration in minced lamb

\section{Model performance comparison}

Once the important wavelengths were selected, the spectral data were reduced to a matrix of 18 samples $\times 7$ variables by selecting those variables at the identified optimum wavelengths. The number of variables was reduced from 237 to 7 . A new PLSR model was also created to verify the potential of selected important wavelengths over the PLSR model developed using the full spectral range. Table 1 displays results of calibration models and cross-validation models performance for heart adulteration in minced lamb using both full wavelengths range and seven selected optimum wavelengths. The prediction ability of PLSR model with selected important wavelength was equivalent to the PLSR model with full spectra, with $R_{C V}^{2}(0.97$ vs. 0.97$)$, and RMSECV $(1.80 \%$ vs. $1.84 \%)$, demonstrating that the selected wavebands were capable enough to represent the full wavebands for the evaluation of heart adulteration.

Table 1. Comparison of the PLS results for predicting adulteration level on minced lamb meat in full-wavelength and selected-wavelength range

\begin{tabular}{|c|c|c|c|c|}
\hline Model based on & $\mathbf{R}^{{ }{ }_{\mathbf{c}}}$ & RMSEC (\%) & $\mathbf{R}^{{ }{ }_{\mathbf{c v}}}$ & RMSECV (\%) \\
\hline Full-wavelength & 0.99 & 1.21 & 0.97 & 1.80 \\
\hline Selected-wavelength & 0.99 & 1.40 & 0.97 & 1.84 \\
\hline
\end{tabular}




\section{Conclusion}

The current study showed that NIR hyperspectral imaging coupled with PLSR analysis was suitable for predicting adulteration in minced lamb meat. Using SPA, seven optimum wavebands were selected to reduce data redundancy without reducing prediction performance. Using these optimum wavelengths, simple, fast and low cost spectral imaging sensor can be designed for adulterate detection in minced meat.

\section{References}

Andr'es, S., Silva, A., Soares-Pereira, A.L., Martins, C., Bruno-Soares, A.M. and Murray, I. 2008. The use of visible and near infrared reflectance spectroscopy to predict beef $\mathrm{M}$. Longissimus thoracis et lumborum quality attributes. Meat Science. 78: 217-224.

Araújo, M.C.U., Saldanha, T.C.B., Galvão, R.K.H., Yoneyama, T., Chame, H.C., and Visani, V. 2001. The successive projections algorithm for variable selection in spectroscopic multicomponent analysis. Chemometrics and Intelligent Laboratory Systems. 57(2): 65-73.

Ariana, D.P. and Lu, R. 2010. Evaluation of internal defect and surface color of whole pickles using hyperspectral imaging. Journal of Food Engineering. 96: 583-590.

Barlocco, N., Vadell, A, Ballesteros, F., Galietta, G., and Cozzolino, D.2006. Predicting intramuscular fat, moisture and WarnerBratzler shear force in pork muscle using NIR spectroscopy. Animal Science. 83:111-116.

Ding, X., Xu, R. 2010. Near-Infrared Spectroscopic Technique for Detection of Beef Hamburger Adulteration._Journal of Agricultural and Food Chemistry. 48: 2193-2198.

EIMasry, G., Wang, N., Vigneault, C., Qiao, J. and EISayed, A. 2008. Early detection of apple bruises on different background colors using hyperspectral imaging. LWT-Food Science and Technology. 41: 337-345

EIMasry, G., Kamruzzaman, M., Sun, D-W, and Allen, P. 2012. Principles and applications of hyperspectral imaging in quality evaluation of agro-food products: a review. Critical Review in Food Science and Nutrition. 52:999-1023.

Goetz, A.F.H., Vane, G., Solomon J.E. and Rock, B.N. 1985. Imaging spectroscopy for earth remote sensing. Science. 228: 1147-1153.

Grau, R., Sánchez, A.J., Girón, J., Iborra, E., Fuentes, A. and Barat, J.M. 2011. Nondestructive assessment of freshness in packaged sliced chicken breasts using SW-NIR spectroscopy. Food Research International. 44:331-337.

Iqbal, A., Sun, D-W and Allen P. 2013. Prediction of moisture, color and pH in cooked, pre-sliced turkey hams by NIR hyperspectral imaging system. Journal of Food Engineering. 117:42-51.

Kamruzzaman, M., Sun, D-W, EIMasry, G. and Allen, P. 2013. Fast detection and visualization of minced lamb meat adulteration using NIR hyperspectral imaging and multivariate image analysis. Talanta. 103:130-136.

Meza-Márquez, O.G., Gallardo-Velázquez, T., Osorio-Revilla, G. 2010. Application of mid-infrared spectroscopy with multivariate analysis and soft independent modeling of class analogies (SIMCA) for the detection of adulterants in minced beef. Meat Science. 86: 511-519.

Morsy, N. and Sun, D-W. 2013. Robust linear and non-linear models of NIR spectroscopy for detection and quantification of adulterants in fresh and frozen-thawed minced beef. Meat Science. 93(2): 292-302.

Naganathan, G.K., Grimes, L.M., Subbiah, J., Calkins, C.R., Samal, A. and Meyer, G.E. 2008. Visible/near-infrared hyperspectral imaging for beef tenderness prediction. Computers and Electronics in Agriculture. 64:225-233.

Nakariyakul, S. and Casasent, D. 2008. Hyperspectral waveband selection for contaminant detection on poultry carcasses. Optical Engineering. 47:087202-9.

Qiao, J., Ngadi, M.O., Wang, N., Gariepy, C. and Prasher, S.O. 2007. Pork quality and marbling level assessment using a hyperspectral imaging system. Journal of Food Engineering. 83:10-16.

Segtnan, V.H., Høy, M., Lundby, F., Narum, B. and Wold, J.P. 2009. Fat distributional analysis in salmon fillets using non-contact near infrared interactance imaging, a sampling and calibration strategy. Journal of Near Infrared Spectroscopy. 17: 247-253.

Sone, I., Olsen, R.L., Sivertsen, A.H., Eilertsen, G. and Heia, K. 2012. Classification of fresh Atlantic salmon (Salmo salar L.) fillets stored under different atmospheres by hyperspectral imaging. Journal of Food Engineering. 109:482-489.

Wu, D., Shi, H., Wang, S., He, Y., Bao, Y., \& Liu, K. 2012. Rapid prediction of moisture content of dehydrated prawns using online hyperspectral imaging system. Analytica Chimica Acta. 726(13): 57-66.

Wu, D., Shi, H., He, Y., Yu, X. and Bao, Y. 2013. Potential of hyperspectral imaging and multivariate analysis for rapid and noninvasive detection of gelatin adulteration in prawn. Journal of Food Engineering. 119(3): 680-686.

Zhao, M., Downey, G., Donnell, C.O. 2014. Detection of adulteration in fresh and frozen beefburger products by beef offal using mid-infrared ATR spectroscopy and multivariate data analysis. Meat Science. 96:1003-1011. 